\title{
LA DIMENSIÓN INSTITUCIONAL DEL COMPORTAMIENTO EMPRESARIAL
}

\author{
Federico Morales Barragán \\ PROIMMSE-IIA \\ UNIVERSIDAD NACIONAL AuTÓNOMA DE MÉXICO
}

\begin{abstract}
RESUMEN
En el artículo se plantea que el debate latinoamericano dedicado al desarrollo local se enriquece mediante la incorporación del análisis institucional del comportamiento empresarial, el cual tiene una larga tradición en las ciencias sociales. En particular se sugiere estudiar aquél a partir de las reglas, normas, concepciones o significados, revelados en la actuación observable de los empresarios. En correspondencia con esta perspectiva, se propone una guía de observación para obtener datos de dicho comportamiento.
\end{abstract}

Palabras clave: comportamiento empresarial, instituciones, desarrollo local. 


\section{ABSTRACT}

This article proposes that the Latin American debate on local development can be enriched by incorporating an institutional analysis of business performance, something that has a long tradition in social sciences. In particular, the author suggests conducting such an analysis on the basis of rules, norms, conceptions and meanings revealed in the observable actions of entrepreneurs. In line with this perspective, an observation guide for obtaining data on this type of performance is suggested.

Key words: business performance, institutions, local development. 


\section{INTRODUCCIÓN}

El estudio de la dimensión institucional del comportamiento empresarial y su relación con el desarrollo local en América Latina ha estado influenciado, en los últimos años, por contribuciones que subrayan el valor de los ambientes o entornos que propician la comunicación y la colaboración entre diferentes tipos de actores vinculados con un territorio. Dichas contribuciones han desplegado sus argumentos mediante el uso de conceptos tales como sistema productivo territorial (Bramanti y Maggione 1997, Maillat y Kebir 1998); medio innovador (Maillat 1996, Ratti et al. 1997); distrito industrial (Cossentino et al. 1996, Belussi y Gottardi 2000), y sistema agroalimentario localizado (Boucher y Del Pozo 2000, Boucher et al. 2003).

Como resultado de aquella influencia, diversas localidades $y$ regiones latinoamericanas se han estudiado desde una perspectiva orientada, casi exclusivamente, a identificar cómo se pueden establecer, o han erigido, aquellas condiciones de comunicación y colaboración (Helmsing 2001, Llorens et al. 2002).

Los doce casos estudiados en este documento no proporcionan evidencia concluyente acerca de nuevas tendencias en la práctica de la planeación local y regional en América Latina. Sin embargo, estos proporcionan indicios de un «nuevo institucionalismo» emergente ... Las nuevas prácticas buscan dar forma a nuevas modalidades de cooperación público-privada, mediante redes tanto horizontales como verticales. La cooperación público-privada se centra en la creación de mesoinstituciones a nivel del territorio y la industria. Ellas involucran una variedad de procesos de aprendizaje, que en algunos casos es más restringido pero en otros alcanza el estado de un «medio innovador» (Helmsing 2001: 13). 
En estudios como el recién citado se sugiere, muchas veces de manera implícita, la existencia de un camino único para promover el desarrollo local, el cual se delinea mediante el uso de los conceptos a los que se ha hecho referencia. Esto es, las condiciones institucionales presentes en las localidades y regiones bajo estudio se muestran como anomalías de una forma genérica construida al margen de valoraciones institucionales precisas, o bien, como ejemplos de prácticas que se aproximan a aquella forma, lo cual parece garantizar su viabilidad.

En contraste, una consideración detallada de las condiciones institucionales de las localidades y regiones bajo estudio revelaría las modalidades posibles de la colaboración, o bien, contribuiría a explicar los elementos específicos que la han hecho posible.

Muchas veces pasa desapercibido que el uso de conceptos tales como sistema productivo territorial, medio innovador, distrito industrial y sistema agroalimentario localizado, ha permitido mostrar el arraigo social y carácter específico de los mecanismos institucionales que propician la colaboración entre diversos actores. Tal es el caso, por ejemplo, de los mecanismos diseñados para estimular el desarrollo de innovaciones en formas de organización económica y social como son los distritos industriales italianos (Morales 2005).

La pertinencia de conceptos tales como sistema productivo territorial, medio innovador, distrito industrial y sistema agroalimentario localizado obedece, precisamente, a su acotamiento espacial y temporal, esto es, a la valoración que con base en éstos se ha hecho de diversos territorios y sus mecanismos institucionales.

Es importante comprender que la necesidad de establecer límites históricos y geográficos a los estudios del comportamiento empresarial no significa sugerir que la teoría es en sí misma irrelevante 
o inútil; historia y teoría no son antitéticas. Más bien, se plantea que tiempo y geografía frecuentemente actúan como delimitaciones fundamentales que necesitan tomarse en cuenta para desarrollar generalizaciones más útiles acerca del comportamiento empresarial (Jones y Wadhwani 2006: 22).

El problema recién expuesto ofrece una motivación para argumentar a favor de revalorar el análisis institucional del comportamiento empresarial en los estudios latinoamericanos dedicados al desarrollo local. Esta cuestión se aborda aquí en dos secciones, primero se recuperan contribuciones de las ciencias sociales que hacen patente el sentido de estudiar la dimensión histórica institucional del comportamiento empresarial; luego se cuestiona la acepción de contexto que frecuentemente se utiliza para estudiar las instituciones y se propone, más bien, aproximarse a ellas en términos de las reglas, normas, concepciones o significados que se manifiestan en el comportamiento observable de los empresarios. Acorde con esta perspectiva, por último se plantea un esquema o guía de observación para obtener datos de dicho comportamiento.

\section{CONTRIBUCIONES DE LAS CIENCIAS SOCIALES ACERCA DEL COMPORTAMIENTO EMPRESARIAL}

En las dos últimas décadas se ha manifestado un renovado interés por las ideas de Joseph Schumpeter que incluso ha conducido al establecimiento de una asociación internacional que lleva su nombre (Hodgson 2005). Paradójicamente, solo unos cuantos estudiosos del comportamiento empresarial hacen uso explícito de sus ideas y aún es menor el número de ellos que se refiere a Schumpeter para analizar el proceso de creación de nuevas empresas. Al respecto, son reveladores algunos resultados de la indagación llevada a cabo por Aldrich para el periodo comprendido entre 1980 y 2004 : 
... en las cinco revistas más importantes dedicadas a la teoría de la organización, hubo 274 menciones del término empresario, pero solo 14 por ciento presentaron co-ocurrencia de los términos empresario y Schumpeter. [De este universo] solo 9 artículos se ocuparon del establecimiento de nuevas empresas ... En las tres revistas más importantes de sociología, Schumpeter fue mencionado en 72 artículos en una amplia variedad de contextos. Empresario se mencionó 94 veces. Solo 10 por ciento de [aquellos artículos] presentó la ocurrencia conjunta de Schumpeter y empresario. De estos, solo dos trataron el asunto de la creación de nuevas empresas (Aldrich 2005: 455, itálicas en el original).

Los resultados anteriores son de interés porque hacen evidente el conocimiento limitado que se tiene de la obra de un autor muchas veces aludido en los estudios sobre el comportamiento empresarial, más aún en los que relacionan este tema con el desarrollo local. No es extraño, por ello, que en general se desconozcan las perspectivas diferentes planteadas por el propio Schumpeter en relación con ese tema. Aldrich (2005) comenta que los escritos de Schumpeter publicados en 1912 y 1928 muestran dos perspectivas distintas de su reflexión acerca de los empresarios. En el primero, cuyo contenido es más conocido, el énfasis se encuentra en las características personales de los empresarios, a quienes se les atribuyen enormes poderes de liderazgo. En cambio, el texto de 1928 se ocupa de la función empresarial, no de la persona que lleva a cabo ésta. La despersonalización de la concepción implica, en esta última perspectiva, que el comportamiento empresarial no puede entenderse al margen de su contexto histórico específico y sus instituciones. «Schumpeter argumentó que el comportamiento empresarial debe ser colocado en su contexto social e histórico ... En esta visión despersonalizada, la clave se encuentra en la 
actividad colectiva de muchas personas, actuando dentro de una época histórica, no en las acciones de individuos particulares» (Aldrich 2005: 455).

Una opinión del propio Schumpeter apunta:

Personalmente creo que existe un incesante intercambio entre el análisis histórico y el teórico y que, aunque para la investigación de cuestiones particulares puede ser necesario atender momentáneamente uno solo de éstos, ninguno de los dos debería de perder de vista la contribución del otro ... En consecuencia nosotros deberíamos formular nuestra tarea como un intento por escribir una historia exhaustiva del comportamiento empresarial (Schumpeter 1949, en Jones y Wadhwani 2006: 7).

El señalamiento de Schumpeter coincide con la opinión expresada por Baumol respecto de la especificidad histórica de las instituciones y el efecto que estas ejercen en el comportamiento de los empresarios. «En la medida que las reglas del juego cambian muy lentamente ... el estudio de casos para investigar mis hipótesis me conduce inevitablemente a ejemplos que se distribuyen a lo largo de distintos periodos y abarcan diferentes culturas y ubicaciones» (Baumol 1990, en Jones y Wadhwani 2006: 23).

Una orientación similar a la de Baumol y Schumpeter, en el texto de 1928, se encuentra presente en autores que formaron parte de la Escuela histórica alemana y plantearon problemas relevantes para el análisis social.

Krabbe (1996) ofrece una documentada revisión de las tradiciones historicista y organicista en la evolución del pensamiento económico, misma que, por supuesto, hace una amplia referencia a miembros y seguidores de la Escuela histórica alemana. Enseguida una cita extensa que ubica la importancia de considerar las contribuciones de algunos autores de 
esta escuela en la discusión en torno al comportamiento empresarial y su dimensión institucional.

Durante el siglo diecinueve, el planteamiento de Adam Smith sobre el laissez faire, «no intervención del gobierno», completó su marcha triunfal por todo el mundo. En Alemania en particular, la población estaba buscando formas más refinadas de pensamiento que pudieran tener mayor sintonía con la realidad económica y los problemas de actualidad de la política económica ... En respuesta a las opiniones prevalecientes aparecieron los trabajos de Wilhelm Roscher, Karl Knies y Bruno Hildebrand, fundadores de la Escuela Histórica. A diferencia de Karl Marx, ellos no rechazaron radicalmente la visión optimista de la visión económica liberal de la Escuela Clásica. Más bien, relativizaron el modelo Clásico y centraron sus críticas en los siguientes puntos. Un enfoque Clásico, como el de David Ricardo, fue considerado marcadamente individualista y legitimador de efectos objetables del egoísmo personal. Además, creyeron que la teoría Clásica reivindicaba erróneamente ser aplicable a todas las etapas del desarrollo económico. La Escuela Histórica intentó evitar tales carencias utilizando una metodología que fue, al menos parcialmente, «organicista» (Krabbe 1996: 1, itálicas en el original).

El rechazo a la pretendida universalidad de las proposiciones del pensamiento clásico condujo a los autores de la Escuela histórica alemana a centrar su atención en los vínculos continuamente cambiantes que se establecen entre los individuos y la sociedad, así como en sus mutuas determinaciones. En ese sentido, destaca que la mayoría de sus miembros sostiene que la economía debe centrarse en el estudio del «es» más que en el del «deber ser» (Krabbe 1996: 18). Explicar por qué suceden las cosas y no cómo deberían ser establece una perspectiva cuyas valoraciones corresponden, necesariamente, a contextos históricos específicos. 
Para nosotros la historia no es un medio, sino el objeto de nuestras investigaciones ... en nuestra teoría nosotros desistimos de la elaboración de ideales. En cambio, nosotros intentamos descripciones simples, primero de la naturaleza y necesidades de las personas, en segundo lugar de las leyes e instituciones [referidas] a las últimas, y finalmente del éxito mayor o menor que se haya logrado a través de estas leyes e instituciones (Roscher 1849, en Hodgson 2001: 59).

Según Hodgson (2001), opiniones como las anteriores sugieren un rechazo de teorías universales en favor de teorías particulares basadas en descripciones de fenómenos específicos, sin embargo, más allá de la discusión acerca de los insumos teóricos y conceptuales que requieren tales descripciones, el mérito de aquellas opiniones es el de apreciar el estudio de las instituciones económicas específicas, en vez de intentar construir explicaciones sobre la base de proposiciones pretendidamente universales.

Knies, al igual que Roscher (Krabbe 1996), considera que la teoría de David Ricardo solo puede aplicarse a ciertas etapas del desarrollo social y señala, también, que junto a la teoría económica existe la «praxis» científica, esto es, el investigador orienta su actividad a la búsqueda tanto de analogías como de rasgos particulares en las situaciones bajo estudio. En ese sentido, Knies plantea complementar la perspectiva deductiva del análisis clásico con un enfoque inductivo para así dar una base empírica a la teoría. «Desde cualquier punto de vista que se considere la teoría económica, es una condición indispensable que esta se base en hechos históricos a través de los cuales dicha teoría pueda ser determinada sin entrar en conflicto con cualquier tipo de veracidad» (Knies 1853, en Krabbe 1996: 29).

El enfoque sugerido por los miembros de la Escuela histórica alemana muestra claramente una concepción más amplia de los fenómenos económicos, la cual incorpora 
explícitamente su dimensión institucional. En Schmoller, por ejemplo, el sistema económico es visto como un sistema compuesto por tres subsistemas articulados, lo cual pone de manifiesto su concepción organicista de aquél. El sistema mental o ámbito de las decisiones está compuesto por los subsistemas ético, de las instituciones y de los institutos; el sistema tecnológico se integra por dos subsistemas, uno referido al conocimiento básico y otro al aplicado; finalmente el sistema del capital y la naturaleza está integrado por el subsistema de la maquinaria y el de los artefactos y la geografía (Krabbe 1996: 66).

Respecto del primer sistema, Schmoller considera que las instituciones son factores parciales del orden social que se manifiestan en costumbres, reglas y leyes. En cuanto a la distinción entre las instituciones y los institutos, este autor opina que las primeras remiten a ideas tales como la propiedad, el matrimonio, la esclavitud, mientras que los institutos representan la contraparte empírica de dichas ideas: si el matrimonio es una institución, la familia es entonces un instituto; una compañía o empresa cualquiera es un instituto y representa la contraparte empírica de la institución empresa privada. Concebidos así, tanto las instituciones como los institutos corresponden, necesariamente, a etapas particulares del desarrollo económico (Krabbe 1996: 61, 65).

Como lo revelan las opiniones de los miembros de la Escuela histórica alemana citados con anterioridad, resulta sumamente limitado estudiar cualquier tipo de proceso económico sin considerar sus condiciones históricas y por tanto sus trasformaciones institucionales. Esta misma perspectiva ha sido compartida a lo largo de varias décadas por diversos autores interesados en el estudio del comportamiento empresarial. Stinchcombe expresa enfáticamente dicha posición al señalar que «... las personas construyen organizaciones que son culturalmente enraizadas e históricamente específicas, lo que refleja 
las condiciones sociales de una coyuntura histórica particular» (Stinchcombe 1965, en Aldrich 2005: 451).

Jones y Wadhwani (2006) también insisten en la importancia de elaborar estudios del comportamiento empresarial recuperando la dimensión histórica de éste. Su argumentación se desarrolla con base en el comentario de tres aspectos específicos: en primer lugar, estos autores subrayan que omitir el estudio del contexto histórico puede conducir a dos tipos de interpretaciones falaces, la más común de éstas se expresa en la generalización arbitraria de explicaciones, en las que se omite cualquier consideración de índole espacial y temporal. Con base en un estudio desarrollado por Kilby (1971) en la zona occidental de África, Jones y Wadhwani ponen de relieve el distinto significado que puede otorgarse al término comportamiento empresarial. En particular, señalan que los hallazgos de Kilby hacen patente las limitaciones de la explicación neoclásica al respecto. En los países subdesarrollados, los recursos necesarios para establecer nuevas empresas no están disponibles mediante mecanismos arquetípicos de mercado, muchas veces tales recursos deben construirse, y en consecuencia estas actividades, que rebasan las tareas de identificación y adquisición de recursos, deben ser consideradas como parte de la actividad empresarial.

Otra posibilidad de incurrir en interpretaciones falaces se manifiesta en opiniones que plantean la necesidad de nuevas bases del análisis social para explicar rasgos considerados inéditos en las formas de organización contemporánea. Un ejemplo de ello es la referencia a la proliferación de empresas que «nacen globales» y la supuesta necesidad de nuevas teorías para su comprensión. 
Estas formas de organización basadas en relaciones reticulares no son en realidad nuevas. Mientras que la mayoría de las empresas de los Estados Unidos se han expandido hacia el exterior de manera paulatina, la estrategia de «nacer global» estaba presente en muchas experiencias empresariales en el siglo diecinueve. En la víspera de la Primera Guerra Mundial, por ejemplo, existieron cientos de compañías británicas que «nacieron globales» y operaron exclusivamente en países extranjeros basadas en sus relaciones con otras empresas. Patrones similares existieron para compañías de muchos países pequeños con mercados locales limitados (Wilkins \& Schroter 1998, en Jones y Wadhwani 2006: 19-20).

Estos autores consideran, con base en el ejemplo anterior, que el conocimiento de la evolución histórica del comportamiento empresarial debería conducir a indagar no sobre la supuesta novedad o carácter inédito de la estrategia de las empresas estadounidenses de «nacer globales», sino sobre las razones que propiciaron el cambio en su modalidad de internacionalización.

Un aspecto adicional considerado por Jones y Wadhwani se refiere a la influencia que ejerce la sucesión de cambios históricos en el comportamiento de los empresarios. Es decir, no solo importa considerar los distintos rasgos que caracterizan los contextos históricos sino también la trayectoria mediante la cual se configuran estos últimos.

A pesar de que las perspectivas evolucionistas del análisis económico han desarrollado métodos para valorar la dependencia respecto de la trayectoria de sucesos (Malerba et al. 2001, entre otros), esta cuestión no ha sido apreciada suficientemente por quienes estudian los procesos de creación de nuevas empresas.

En las últimas dos décadas se ha prestado cada vez menos atención a comprender el contexto histórico del comportamiento empresarial, el asunto resulta asombroso si se toma en cuenta la 
posición ampliamente difundida en la literatura teórica respecto de la importancia de comprender las necesidades de las iniciativas empresariales como un proceso dinámico operando en contextos específicos (Jones y Wadhwani 2006: 14, itálicas en el original).

Según Jones y Wadhwani esta omisión obedece al supuesto, poco fundamentado, de que las empresas establecidas o consolidadas están más expuestas a la influencia de la trayectoria de sucesos. Como ellos mismos señalan, la identificación de nuevas oportunidades y la comprensión de sus condiciones de éxito o fracaso son aspectos que forman parte de un proceso difícilmente aprensible mediante la sola valoración de acontecimiento ubicados en el presente.

Finalmente, Jones y Wadhwani (2006) mencionan que la comprensión de otros factores históricos presentes en la actividad empresarial, más allá del ámbito de la elecciones que hacen los empresarios a lo largo del tiempo, requiere del aporte de quienes asumen la perspectiva del institucionalismo histórico.

[Este] tiende a centrar más su atención en el estudio de cómo se forman las reglas que frecuentemente están enraizadas en estructuras sociales históricas que no son fácilmente identificables cuando uno sólo dirige su atención al proceso de elección de los agentes ... A pesar de la prevalencia del «institucionalismo histórico» en las corrientes actualmente dominantes de las ciencias sociales, ha habido relativamente poco trabajo en esta vertiente por parte de los científicos sociales interesados en el tema del comportamiento empresarial (Jones y Wadhwani 2006: 26-27).

Como ha podido apreciarse en esta sección, la valoración histórica y por tanto institucional del comportamiento empresarial constituye una preocupación de larga data en el análisis 
social. Enseguida se aborda un problema específico de aquélla, el cual tiene que ver con el papel de las instituciones en el proceso de creación de las empresas.

\section{INSTITUCIONES Y CREACIÓN DE EMPRESAS: CONSIDERACIONES EN TORNO A UN MARCO DE INTERPRETACIÓN}

El rasgo distintivo del comportamiento empresarial es todavía objeto de debate (Aldrich 2005). Algunos consideran que la función más relevante de los empresarios se refiere a la posibilidad de introducir innovaciones; otros mencionan la capacidad de identificar oportunidades u opciones de negocios; también existen opiniones, como la de Gartner, que asocian el comportamiento empresarial a la creación de empresas. «[El] comportamiento empresarial debe ser estudiado concentrándose en el comportamiento y actividades de las personas tratando de crear empresas, más que en sus estados psicológicos y características personales» (Gartner 1988, en Aldrich 2005: 457).

En la discusión que sigue se adopta esta última perspectiva y se reflexiona en torno al papel que juegan las instituciones en el proceso de creación de las empresas; en particular, se cuestiona la acepción de contexto que frecuentemente se utiliza para estudiar la dimensión institucional del comportamiento empresarial y más bien se propone aproximarse a ésta en términos de las reglas, normas, concepciones o significados que se manifiestan en el comportamiento observable de los empresarios. Acorde con dicha perspectiva se plantea un esquema o guía de observación para obtener datos de tal comportamiento, esquema construido con base en orientaciones compartidas por Scott (2001) y Meriggi y Rossi (s/a). ${ }^{1}$ 
Bajo el entendido de que resulta limitado analizar el comportamiento empresarial al margen de sus condiciones histórico institucionales, la incorporación de estas en el mencionado análisis exige hacer explícita la concepción de las instituciones que se utiliza.

De acuerdo con Scott (2001), las instituciones son estructuras sociales construidas por tres tipos de sistemas: los de regulación, los normativos, y los culturales-cognitivos. Según este autor, dichos sistemas han sido considerados por los investigadores sociales, independientemente de sus enfoques particulares, como los ingredientes fundamentales o los pilares de las instituciones. Estos pilares se encuentran, a su vez, insertos en mecanismos de trasmisión constituidos por sistemas simbólicos, sistemas relacionales, rutinas y cierto tipo de artefactos. La consideración conjunta de los pilares de las instituciones como sus mecanismos de trasmisión permite generar una clasificación de estos últimos, como la que se aprecia en la tabla siguiente.

Tabla 1. Pilares y mecanismos de trasmisión institucionales

\begin{tabular}{|c||c|c|c|}
\hline \multicolumn{1}{|c||}{$\begin{array}{c}\text { Mecanismos de } \\
\text { trasmisión }\end{array}$} & Regulación & Normativo & Cultural-cognitivo \\
\cline { 2 - 4 } & Reglas, leyes & Valores, expectativas & $\begin{array}{c}\text { Categorías, } \\
\text { tipificaciones, } \\
\text { esquemas }\end{array}$ \\
\hline $\begin{array}{c}\text { Sistemas } \\
\text { simbólicos }\end{array}$ & $\begin{array}{c}\text { Sistemas de gobernanza, } \\
\text { sistemas de poder } \\
\text { relacionales }\end{array}$ & $\begin{array}{c}\text { Regímenes, sistemas de } \\
\text { autoridad }\end{array}$ & $\begin{array}{c}\text { Isomorfismo } \\
\text { estructural, identidades }\end{array}$ \\
\hline Rutinas & $\begin{array}{c}\text { Procedimientos estandarizados } \\
\text { de operación }\end{array}$ & $\begin{array}{c}\text { Trabajos, funciones, } \\
\text { obediencia a deberes }\end{array}$ & Guiones \\
\hline Artefactos & $\begin{array}{c}\text { Objetos que cumplen con } \\
\text { especificaciones establecidas }\end{array}$ & $\begin{array}{c}\text { Objetos donde se materializan } \\
\text { convenciones, estándares }\end{array}$ & $\begin{array}{c}\text { Objetos que poseen un } \\
\text { valor simbólico }\end{array}$ \\
\hline
\end{tabular}

Fuente: Scott (2001: 77).

La distinción entre los pilares institucionales expuestos en la tabla resulta necesaria, pero no niega la estrecha relación que existe entre los tres tipos de sistemas que constituyen aquéllos ni su retroalimentación con el comportamiento social, pues como señala Scott, 
«[las] reglas, normas y significados surgen en la interacción, y ellos son preservados y modificados por el comportamiento humano» (Scott 2001: 49).

La concepción de las instituciones como estructuras sociales de regulación subraya la restricción que estas ejercen sobre el comportamiento humano; en cambio, la dimensión normativa de las instituciones pone de relieve que las normas no solo limitan el comportamiento social sino también lo hacen posible y lo fortalecen. En este sentido, como subraya Hodgson (2001), las instituciones restringen o limitan las actividades de las personas, y también promueven la adopción de nuevos comportamientos. En cuanto al componente cultural-cognitivo de las instituciones, este es sintetizado claramente por Berger y Kellner: «[c]ada institución humana es, como sea, una sedimentación de significados o, para variar la imagen, una cristalización de significados en forma objetiva» (1981: 31, en Scott 2001: 57). O bien, como señala el propio Scott haciendo referencia a quienes postulan un enfoque cultural-cognitivo, «... la conformidad ocurre en muchas circunstancias porque otros tipos de comportamiento son inconcebibles; las rutinas se siguen porque se da por sentado que "es la forma en que nosotros hacemos estas cosas"» (Scott 2001: 57).

Respecto de los mecanismos de trasmisión, los elementos ubicados en las casillas de la Tabla 1 constituyen formas evidentes de su expresión, por ello, su significado específico no requiere mayor aclaración, salvo el sentido que se da al término «isomorfismo estructural». En este contexto, dicho término se refiere al hecho de que muchas formas de relación son compartidas ampliamente por múltiples organizaciones, dicho de otro modo, las formas son similares en una gran variedad de ámbitos organizativos.

El marco conceptual anterior, que integra los sistemas de regulación, los normativos y los culturales-cognitivos con los mecanismos de trasmisión mediante los cuales se 
expresan aquéllos, esclarece la afirmación, ya habitual, de que una organización está profundamente incrustada en un ambiente institucional, pues evidencia que

[una] organización está apoyada y constreñida por fuerzas institucionales [e] incorpora dentro de sus propios límites una multitud de características institucionalizadas, bajo la forma de culturas, sistemas relacionales, rutinas y artefactos. [De ahí] que es apropiado hablar del grado en que los componentes o características organizacionales están institucionalizados (Scott 2001: 82).

Los elementos de la tabla anterior establecen un marco de referencia útil para valorar el papel de las instituciones en el proceso de creación de las empresas. Sin embargo, dichos elementos pueden ser utilizados bajo dos ópticas distintas: una que los entiende como el contexto donde ocurren las relaciones sociales y subraya entonces la determinación o influencia que ejercen las instituciones en el comportamiento de las personas; y otra en la que no se privilegia ninguna de las influencias y por tanto se busca indagar la mutua relación entre las instituciones y el comportamiento humano. Esta perspectiva, como se ha visto en la sección precedente, ha estado presente desde hace muchos años en las discusiones referidas al comportamiento empresarial.

Una implicación metodológica de la perspectiva señalada es que las instituciones no se conciben como un elemento yuxtapuesto al comportamiento empresarial, es decir, si aquellas son vistas como un marco de referencia o contexto, el problema metodológico se plantea en términos de cómo integrar éste en la valoración del comportamiento de los empresarios. Así es, por ejemplo, la orientación que asumen algunos autores adscritos al análisis neoinstitucional convencional (Williamson 1999, Furubotn y Richter 2000, entre otros). 
En la visión que sostiene la necesidad de valorar las influencias mutuas entre individuos e instituciones, dichas influencias aparecen como una parte constitutiva de cualquier relación social, no como un componente yuxtapuesto. ${ }^{2}$

Meriggi y Rossi (s/a) plantean una orientación clave para instrumentar el enfoque precedente al afirmar que el comportamiento observable de los empresarios revela los rasgos del contexto social donde aquél se sitúa. ${ }^{3}$ Esto es, a diferencia de una estrategia de análisis que parte primero de identificar las virtudes o carencias de un contexto institucional con base en los rasgos de un tipo ideal de este último, y luego supone la forma en que dichos rasgos afectan el comportamiento empresarial; la estrategia que se desprende del señalamiento de Meriggi y Rossi exige, como punto de partida, caracterizar la evolución de los comportamientos empresariales observados para poder apreciar en qué forma los empresarios procesan, a lo largo de un periodo determinado, los condicionamientos emanados de las instituciones involucradas en sus relaciones, y también los cambios que se registran en ellas. Bajo esta óptica, y merece insistirse en ello, las instituciones no aparecen yuxtapuestas al comportamiento de los empresarios, como si en algún momento previo las mencionadas pudieran existir en un ámbito ajeno al de las relaciones sociales; por el contrario, es a través del comportamiento empresarial que las instituciones revelan su historicidad en este ámbito particular de los procesos sociales.

Paradójicamente, en el reporte de Meriggi y Rossi al que se ha hecho referencia, la influencia de las instituciones sobre ciertos comportamientos empresariales se concibe en términos de un contexto; en particular, dichos autores comparan rasgos de lo que ellos llaman ambientes institucionales de Italia y Estados Unidos, y comentan sus efectos en las prácticas de dirección de las empresas y el diseño de sus estrategias. No obstante esta perspectiva, que a mi juicio resulta contradictoria con la orientación precedente planteada 
por los mismos autores, aquí interesa identificar los ámbitos institucionales que Meriggi y

Rossi consideran en su análisis, pues aquéllos son retomados más adelante.

Para Meriggi y Rossi (s/a), los ámbitos institucionales relevantes en las prácticas de dirección de las empresas y el diseño de sus estrategias son los siguientes: el papel del gobierno, el mercado financiero, el sistema educativo, la cultura, y el sistema legal.

Respecto del papel del gobierno, la atención se centra en valorar su grado de intervención mediante políticas de fomento industrial y, en particular, si dichas políticas estimulan la adaptación de las empresas. En relación con el mercado financiero se identifica su dimensión, el grado de liquidez de los instrumentos que ofrece y el control que estos ejercen sobre el funcionamiento de las empresas. En cuanto al sistema educativo se considera su grado de centralización y homogeneidad, así como si promueve el ascenso por logro de méritos y si está orientado a la colocación en el mercado de trabajo. La consideración de los aspectos culturales se restringe a si estos fomentan la iniciativa individual. Finalmente, del sistema legal solo se toman en cuenta las disposiciones en torno a las situaciones de quiebra de la empresa.

La discusión sostenida en esta sección plantea, entre otros, el problema de diseñar métodos para estudiar la dimensión institucional del comportamiento empresarial, coherentes con la perspectiva de estudiar las instituciones no como un contexto sino como las reglas, normas, concepciones o significados, involucrados en el comportamiento observable de los empresarios. Con la finalidad de ilustrar una forma de encarar este problema, enseguida se presenta un esquema o guía de observación que recupera los siguientes elementos: primero, la concepción de Scott (2001) acerca de las instituciones; segundo, los ámbitos institucionales propuestos por Meriggi y Rossi (s/a), y el señalamiento de estos últimos respecto de que el comportamiento observable de los empresarios revela los rasgos de su dimensión institucional. 
Tabla 2. Dimensión institucional del comportamiento empresarial

\begin{tabular}{|c|c|c|c|c|c|}
\hline \multirow{3}{*}{$\begin{array}{c}\text { Ámbitos donde se manifiesta el } \\
\text { comportamiento empresarial }\end{array}$} & \multicolumn{5}{|c|}{ Dimensión institucional } \\
\hline & \multicolumn{3}{|c|}{ Reglas } & \multirow{2}{*}{$\begin{array}{c}\text { Normas } \\
\text { Sistema } \\
\text { educativo (4) }\end{array}$} & \multirow{2}{*}{$\begin{array}{l}\text { Concepciones } \\
\text { y significados } \\
\text { Aspectos } \\
\text { culturales (5) }\end{array}$} \\
\hline & $\begin{array}{c}\text { Papel del } \\
\text { gobierno (1) }\end{array}$ & $\begin{array}{l}\text { Sistema } \\
\text { legal (2) }\end{array}$ & $\begin{array}{c}\text { Mercado } \\
\text { financiero (3) }\end{array}$ & & \\
\hline \multicolumn{6}{|l|}{ Origen de la iniciativa } \\
\hline \multicolumn{6}{|l|}{ Capacidades y recursos propios } \\
\hline \multicolumn{6}{|l|}{ Relación con otros actores } \\
\hline Reglas y funciones internas & & & & & \\
\hline
\end{tabular}

(1) Políticas de fomento económico.

(2) Figuras jurídicas adecuadas para promover la creación de empresas.

(3) Disponibilidad de instrumentos financieros adecuados al perfil empresarial.

(4) Generación de hábitos de trabajo.

(5) Elementos que favorecen o inhiben la disposición a emprender.

Fuente: elaboración con base en Scott (2001) y Meriggi y Rossi (s/a).

En las filas de la tabla anterior se identifican ámbitos donde se manifiestan acciones observables relacionadas con el propósito de establecer nuevas empresas. Estos ámbitos son similares a las definiciones y generalizaciones empíricas expuestas por Aldrich (2005: 459), relacionadas con la investigación sobre la creación de empresas.

A su vez, el desglose de la dimensión institucional que aparece en las columnas recupera los elementos considerados en la propuesta de Meriggi y Rossi delimitados a aspectos específicos que expresan reglas, normas y concepciones o significados; de manera que la influencia de las instituciones en el proceso de creación de empresas exige considerar la forma en que aquellas se manifiestan en el comportamiento de los empresarios. Debe subrayarse, además, que dicho comportamiento no se concibe como algo homogéneo; la delimitación establecida en las filas de la Tabla 2 permite distinguir la influencia particular de reglas, normas, concepciones y significados en diversas facetas del comportamiento empresarial. Así lo revelan algunos resultados de indagaciones llevadas a cabo por Morales (2007) con base en este esquema o guía de observación, algunos de los 
cuales son similares a los expuestos en Aldrich (2005: 458 y ss). En ambos casos destaca, por ejemplo, la importancia de los recursos propios y la experiencia laboral previa en el establecimiento de nuevas empresas.

Respecto del primero, se hace referencia a los diversos condicionamientos que resultan de los términos onerosos establecidos por instancias crediticias. Respecto de la última, Aldrich menciona su contribución en tres aspectos específicos: los vínculos dentro y fuera de las organizaciones; el hecho de que la nueva empresa normalmente está relacionada con el giro o actividad previa; y por último la experiencia en términos de prácticas, valores, vocabularios e identidades que se adaptan al nuevo entorno representado por la empresa (Aldrich 2005: 459).

No es el propósito de este artículo presentar con detalle los resultados emanados de la aplicación de la guía de observación propuesta en la Tabla 2. Es evidente que la discusión sobre esta información dará lugar a nuevas adecuaciones del instrumento. La presentación de éste solo pretende ilustrar una forma de aproximarse al estudio de la dimensión institucional del comportamiento empresarial que busca ser coherente con la argumentación sostenida en esta segunda sección del artículo.

\section{COMENTARIOS FINALES}

El análisis institucional del comportamiento empresarial forma parte de una larga tradición en las ciencias sociales que es necesario recuperar en el debate latinoamericano en torno al desarrollo local. Las políticas de fomento económico asociadas a éste no pueden omitir la valoración de la especificidad histórica de las instituciones. Dicha valoración exige un uso acotado, temporal y espacial, de conceptos tales como sistema productivo territorial, medio 
innovador, distrito industrial, y sistema agroalimentario localizado, todos ellos de uso frecuente en los estudios en torno al desarrollo local.

El análisis institucional del comportamiento empresarial también exige valorar la mutua relación que se establece entre las instituciones y el comportamiento de los empresarios. Documentar esta relación requiere concebir las instituciones como parte inherente de las relaciones sociales, no como un componente yuxtapuesto a éstas. Por ello se propone estudiar las instituciones no como un contexto sino teniendo en cuenta las reglas, normas, concepciones o significados que se revelan en el comportamiento observable de los empresarios. 


\section{BIBLIOGRAFÍA}

Aldrich, Howard E., 2005, «Entrepreneurship». En The Handbook of Economic Sociology, editado por Neil J. Smelser y Richard Swedberg, pp. 451-477. Princeton University Press, Princeton, New Jersey.

Belussi, Fiorenza y Giorgio Gottardi (eds.) 2000, Evolutionary Patterns of Local Industrial Systems. Towards a cognitive approach to the industrial district. Ashgate, Aldershot.

Boucher, François y Luz Mariela del Pozo, 2000, Modulo de capacitación sobre Sistemas Agroalimentarios Localizados. SIAL, publicación en línea: http://www.ciat.cgiar.org/agroempresas/espanol/Rec_de_info/memoriasiicurs/cd_cu rso/Contenido/Modulo\%203/Submodulos\%203.3/modulo_sial.pdf [consulta: octubre de 2007].

Boucher, François et al., 2003, Agro-industrie rurale et lutte contre la pauvreté: les Systèmes Agroalimentaires Localisés contribuent-ils au enforcement des capabilités? Paris, en línea: http://afm.cirad.fr/documents/5_Agro_industries/CD_AFM/textes/528.pdf [consulta: octubre de 2007].

Bramanti, Alberto y Mario Maggioni (eds.), 1997, La dinamica dei sistemi produttivi territoriali: teorie, tecniche, politiche. Franco Angeli, Milano.

Cossentino, Francesco et al., 1996, Local and regional response to global pressure: The case of Italy and its industrial districts. International Institute of Labour Studies, Geneva.

Furubotn, E. y R. Richter, 2000, Institutions and Economic Theory. The Contribution of New Institutional Economics. University of Michigan, Ann Arbor. 
Helmsing, A. H. J., 2001, Partnerships, Meso-institutions and Learning New local and regional economic development initiatives in Latin America. Institute of Social Studies, The Hague, The Netherlands, publicación en línea: http://www1.worldbank.org/wbiep/decentralization/library15/Helmsing.pdf [consulta: enero de 2002].

Hodgson, Geoffrey M., 2001, How Economics forgot History. The Problem of Historical Specificity in Social Sciences. Routledge, London.

2005, «Introduction». En A Modern Reader in Institutional and Evolutionary Economics, pp. xiii-xxix, editado por Geoffrey M. Hodgson. European Association for Evolutionary Political Economy, Edward Elgar, Chaltenham, UK.

Jones, Geoffrey y R. Daniel Wadhwani, 2006, «Schumpeter’s Plea: Rediscovering History and Relevance in the Study of Entrepreneurship». Working Papers, Harvard Business School, July, publicación en línea: http://www.hbs.edu/research/pdf/06036.pdf [consulta: octubre de 2007].

Krabbe, J. J., 1996, Historicism and Organicism in Economics: The Evolution of Thought. Kluwer Academic Publishers, Dordrecht.

Llorens, Juan Luis et al., 2002, Estudios de caso de desarrollo económico local en América Latina. Serie de informes de buenas prácticas del Departamento de Desarrollo Sostenible. Banco Interamericano de Desarrollo Washington, D.C., publicación en línea: http://www.economia.gob.mx/pics/p/p2761/cipi_1Adeseconomicolocalbid.pdf [consulta: enero de 2003].

Maillat, D., 1996, Du district industriel au milieu innovateur: contribution a une analyse des organisations productives territorialisées. IRER, Neuchâtel. publicación en línea: http://www.unine.ch/irer/wp9606.doc [consulta: julio de 1998]. 
Maillat, D. y Laila Kebir, 1998, Learning region et systèmes territoriaux de production. Neuchâtel, IRER, publicación en línea: http://www.unine.ch/irer/wp9802b.doc [consulta: enero de 1999].

Malerba, Franco et al., 2001, «Product Diversification in a "History Friendly" Model of the Evolution of the Computer Industry». En Dynamics of Organizations. Computational Modeling and Organization Theories, pp. 349-375, editado por A. Lomi and E. E. Larsen. American Association for Artificial Inteligence, The MIT Press, Menlo Park, California.

Meriggi, Guido y Alessandro Rossi, s/a, «L'influenza dell'ambiente istituzionale sulle pratiche direzionali e le strategie aziendali e gli effetti sullo sviluppo delle imprese in Italia». Corriere della Sera, publicación en línea: http://www.corriere.it/speciali/american_business/pop-arch-081001.shtml [consulta: marzo de 2007].

Morales, Federico, 2005, «Instituciones e innovación: la experiencia del Grupo K'NAN $\mathrm{CHOCH}$ en Chiapas, México». Revista Europea de Estudios Latinoamericanos y del Caribe, núm. 79, pp. 69-84, octubre. CEDLA, The Netherlands.

2007, «Ámbitos institucionales y comportamiento empresarial». 2. ${ }^{\circ}$ Coloquio Internacional sobre Desarrollo Local "Sistemas locales: capitales, redes e innovación". UAMI-PROIMMSE-IIA-UNAM, Universidad Michoacana de San Nicolás de Hidalgo, México, D. F.

Ratti, Remigio et al. (eds.), 1997, The Dynamics of Innovative Regions. The GREMI Approach. Ashgate, Aldershot.

Scott, W. Richard, 2001, Institutions and Organizations. Sage, Thousand Oaks. 
Revista Pueblos y Fronteras digital

Iniciativa empresarial y desarrollo local en América Latina
Núm. 6, Diciembre 2008 - Mayo 2009

http:// www.pueblosyfronteras.unam.mx

Williamson, Oliver, 1999, The Mechanisms of Governance. Oxford University Press, New York. 


\section{Notas}

${ }^{1}$ Parte de esta discusión también se encuentra en el capítulo de Morales: «Comportamiento empresarial y ambiente institucional: experiencias en la industria chiapaneca de alimentos y bebidas», el cual forma parte del libro colectivo coordinado por Rocío Rosales y Pablo Chauca que será publicado próximamente.

2 En esta argumentación conviene tener presente la distinción entre los términos yuxtaposición e intususcepción. Este último se refiere al «Modo de crecer los seres orgánicos por los elementos que asimilan interiormente, a diferencia de los inorgánicos, que solo crecen por yuxtaposición» (Diccionario de la Lengua Española, vigésima segunda edición, en línea: http://buscon.rae.es/draeI/).

${ }^{3}$ Al respecto, no está de más recordar de nuevo el señalamiento de Stinchcombe, «... las personas construyen organizaciones que son culturalmente enraizadas e históricamente específicas, lo que refleja las condiciones sociales de una coyuntura histórica particular» (Stinchcombe 1965, en Aldrich 2005: 451).

Fecha de recepción: 27 de febrero de 2008

Fecha de aceptación: 8 de abril de 2008 